\section{(D) Check for updates}

Cite this: Dalton Trans., 2020, 49 1591

Received 28th November 2019, Accepted 8th January 2020

DOI: 10.1039/c9dt04572d rsc.li/dalton

\title{
Atomic/molecular layer deposition and electrochemical performance of dilithium 2-aminoterephthalate $\uparrow$
}

\author{
Juho Heiska, (D) Mikko Nisula, Eeva-Leena Rautama, Antti J. Karttunen and \\ Maarit Karppinen (D) *
}

\begin{abstract}
Control of the redox potential of lithium terephthalate $\mathrm{Li}_{2} \mathrm{TP}$ anode material is demonstrated by functionalizing its terephthalate backbone with an electron-donating amino group; this lowers - as intended - the redox potential of $\mathrm{Li}_{2} \mathrm{TP}$ by $0.14 \mathrm{~V}$. The two $\mathrm{Li}$-organic electrode materials, $\mathrm{Li}_{2} \mathrm{TP}$ and $\mathrm{Li}_{2} \mathrm{TP}-\mathrm{NH}_{2}$, are fabricated as crystalline thin films from gaseous precursors using the atomic/molecular layer deposition (ALD/ MLD) technique. The amino-functionalized material possesses a previously unknown crystal structure, addressed here by applying the USPEX evolutionary algorithm for the structure prediction and then LeBail fitting of the experimental XRD pattern based on the predicted structure model. The ALD/MLD fabrication yields in situ lithiated active electrode materials without any conductive additivies or binders and thus allows a straightforward evaluation of their intrinsic electrochemical properties. Comparison between $\mathrm{Li}_{2} \mathrm{TP}$ and its amino-functionalized derivative reveals inferior capacity retention and rate capability characteristics for the latter, which somewhat counterveils the pros-and-cons balance between the two Liorganic electrode materials. From galvanostatic cycling experiments and post-mortem XRD and SEM analysis, the issue with $\mathrm{Li}_{2} \mathrm{TP}-\mathrm{NH}_{2}$ is revealed to be in the morphology changes occurring during the discharge/charge cycling.
\end{abstract}

\section{Introduction}

Lithium-ion batteries have found their way to a diverse range of portable applications owing to their superior performance, in particular regarding the specific energy and efficiency. However, it is well known that the present battery chemistries possess some safety issues; there are also concerns related to the availability of their raw materials and the notable $\mathrm{CO}_{2}$ emissions during their manufacturing. ${ }^{1}$ Alternative next-generation battery chemistries are thus continuously searched for; they should be based on cheap and abundant elements, and exhibit competitive electrochemical performance while being easy to recycle. Organic electrode materials tick many of these requirements and they have been gaining increasing attention in recent years. ${ }^{2,3}$ There are however several issues to be addressed, related e.g. to the fact that small organic molecules suffer from drastic dissolution in the conventional liquid electrolytes, which results in a capacity decay upon cycling. Another challenge is their intrinsically low electronic conductivity.

Department of Chemistry and Materials Science, Aalto University, FI-00076 Espoo, Finland.E-mail: maarit.karppinen@aalto.fi

$\dagger$ Electronic supplementary information (ESI) available. See DOI: 10.1039/ c9dt04572d
Different ways have been adopted to fight these challenges such as coating, grafting, chemical modification, and polymerization.

Organic molecules are remarkably flexible for different chemical modifications, which opens up enormous possibilities to tune the properties of organic electrode materials. ${ }^{4} \mathrm{~A}$ typical organic electrode material comprises one or more redox active functional groups connected to a larger conjugated backbone. The type of the functional group is the determining factor of the potential where the redox reactions occur. The resultant loss or gain of electron(s) is then stabilized by the conjugated backbone. Here we focus on a carbonyl-type material for which upon reduction, the electron is added to the lowest unoccupied molecular orbital (LUMO).

When an additional electron-donating functional group is attached to the carbon skeleton it causes the LUMO energy to become more positive, thereby lowering the redox potential. ${ }^{5} \mathrm{~A}$ somewhat linear relationship between the redox potential and LUMO energy has been reported. ${ }^{6}$ The challenge in the evaluation of the effects of different functional groups is to find simple model systems with a minimum number of other variables. ${ }^{7}$ The present study was inspired by our recent success in depositing high-quality lithium terephthalate $\left(\mathrm{Li}_{2} \mathrm{TP}\right)$ thin films using the atomic/molecular layer deposition (ALD/MLD) technique, and testing these films as a negative electrode 


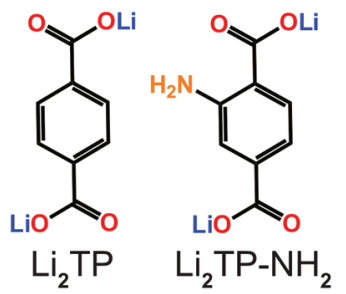

Fig. 1 Molecular structures of $\mathrm{Li}_{2} \mathrm{TP}$ and $\mathrm{Li}_{2} \mathrm{TP}-\mathrm{NH}_{2}$.

material in a pristine form, e.g. without any conductive additives or binder material. ${ }^{8,9}$ The ALD/MLD technique for metalorganics is a strongly emerging branch of the conventional atomic layer deposition (ALD) technology for inorganic thin films, which has been in industrial use already for decades. ${ }^{10,11}$ Like ALD, the combined ALD/MLD technique for metal-organic thin films is based on sequential, self-saturating surface reactions of gaseous precursors and has the capacity to yield thin films with incontestable thickness control and uniformity. ${ }^{12}$ The recent developments in the field of ALD/MLD have opened new avenues towards new interesting metal-organic materials. ${ }^{8,13-18}$ Here we extend the ALD/MLD material repertoire to dilithium 2-aminoterephthalate $\left(\mathrm{Li}_{2} \mathrm{TP}-\mathrm{NH}_{2}\right)$, which then allows us to evaluate the role of the electron-donating amino group on the redox potential of the terephthalate skeleton, with the anticipation that the somewhat high redox potential of $\mathrm{Li}_{2} \mathrm{TP}$ could be decreased by introducing the additional amino group (Fig. 1). Prior to the present work, $\mathrm{Li}_{2} \mathrm{TP}-\mathrm{NH}_{2}$ has been synthesized in bulk form and found to be electroactive but not significantly different from $\mathrm{Li}_{2} \mathrm{TP}$ regarding the redox potential. ${ }^{19}$ On the other hand, these carboxylates behave similarly in sodium ion batteries, ${ }^{20-23}$ and the sodium equivalent $\mathrm{Na}_{2} \mathrm{TP}-\mathrm{NH}_{2}$ has shown the expected decrease in redox potential in bulk samples. ${ }^{24}$

\section{Results and discussion}

\section{ALD/MLD process development}

We have previously developed a facile ALD/MLD process for $\mathrm{Li}_{2} \mathrm{TP}$ films based on $\mathrm{Li}($ thd) and terephthalic acid (TPA) pre- cursors. ${ }^{8}$ Here we adopted the same lithium precursor for the $\mathrm{Li}_{2} \mathrm{TP}-\mathrm{NH}_{2}$ process and replaced the organic precursor to its amino-substituted version, i.e. 2-aminoterephthalic acid (TPA- $\mathrm{NH}_{2}$ ), to make the comparison straightforward. First, we confirmed the most important characteristics of an ideal ALD/ MLD process, that is, the saturation of the so-called growth-percycle (GPC) value (calculated from the XRR-determined film thickness value) with increasing precursor pulse lengths; these experiments were carried out at the deposition temperature $200{ }^{\circ} \mathrm{C}$, and the number of ALD/MLD cycles was fixed to 100 (Fig. 2). In all our depositions the $\mathrm{N}_{2}$ purging time was kept constant, at $4 \mathrm{~s}$ after the $\mathrm{Li}$ (thd) pulse and at $30 \mathrm{~s}$ after the organic precursor pulse. It was revealed that for both the $\mathrm{Li}_{2} \mathrm{TP}-\mathrm{NH}_{2}$ and $\mathrm{Li}_{2} \mathrm{TP}$ processes the film growth saturated with a relatively short (4 s) Li(thd) pulse time, while the organic precursors required a little longer pulsing time, i.e. $6 \mathrm{~s}$ for TPA- $\mathrm{NH}_{2}$ and $10 \mathrm{~s}$ for TPA. The resultant GPC values were appreciably high, and also quite similar for the two processes, i.e. $3.6 \AA$ per cycle for $\mathrm{Li}_{2} \mathrm{TP}-\mathrm{NH}_{2}$ and $3.0 \AA$ per cycle for $\mathrm{Li}_{2}$ TP. Also, in both cases the growth rate decreased significantly with increasing deposition temperature (Fig. 2b); this is a trend seen for most of the reported ALD/MLD processes. ${ }^{8,14,15,17,18,25,26}$

We also confirmed the linearity of the film growth with an increasing number of ALD/MLD cycles after a small incubation period in the beginning (Fig. 2c). The precise determination of the film thickness with XRR was possible for the thinner films only; for the thicker films the surface roughness increased such that the XRR method became less reliable. ${ }^{27}$ From the AFM data the mean surface roughness of the film with 100 cycles was determined to be $\sim 3 \mathrm{~nm}$, while for the thicker films with 200 and 400 ALD/MLD cycles the roughness was $\sim 10$ and $\sim 14 \mathrm{~nm}$, respectively. For the thickest films, we employed spectroscopic ellipsometry for the thickness determination. Additionally, an estimation of the film thickness $(\sim 80 \mathrm{~nm})$ for a film deposited with 200 ALD/MLD cycles was obtained using AFM; a part of the film was scratched (with a surgical knife) and then the surface was scanned with an AFM tip to probe the depth difference between the film surface and the silicon substrate surface (Fig. 2d).

The $\mathrm{Li}_{2} \mathrm{TP}-\mathrm{NH}_{2}$ films were all crystalline after $200 \mathrm{ALD} / \mathrm{MLD}$ cycles. In Fig. 3a we show a part of the GIXRD pattern around
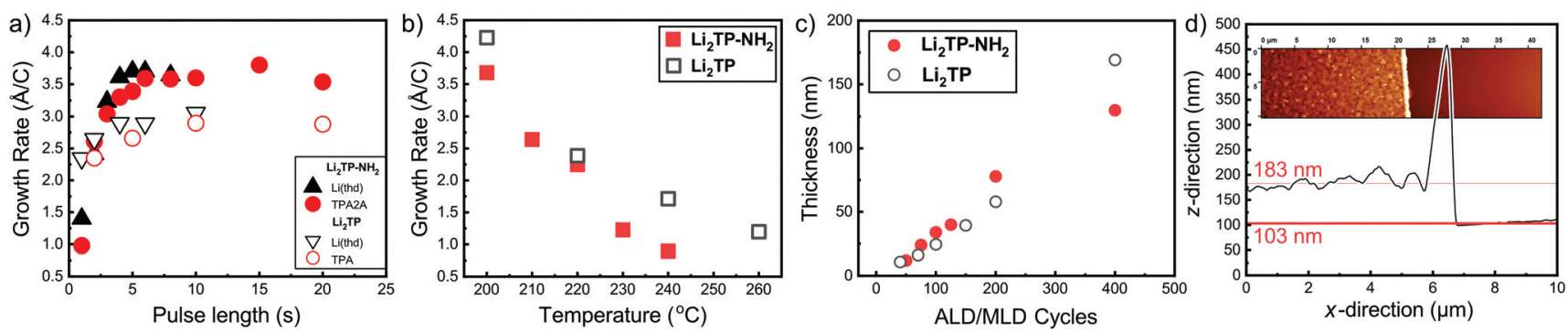

Fig. 2 Growth-per-cycle (GPC) values for the $\mathrm{Li}($ thd $)+T P A-\mathrm{NH}_{2}$ and $\mathrm{Li}($ thd $)+\mathrm{TPA}^{8}$ processes as a function of (a) precursor pulse lengths (at $200{ }^{\circ} \mathrm{C}$ with $100 \mathrm{ALD} / \mathrm{MLD}$ cycles), and (b) deposition temperature with the following precursor pulse lengths: $4 \mathrm{~s}$ of Li(thd) and $10 \mathrm{~s}$ of organic in both cases. In (c) thicknesses of $\mathrm{Li}_{2} \mathrm{TP}-\mathrm{NH}_{2}$ and $\mathrm{Li}_{2} \mathrm{TP}$ films with increasing number of ALD/MLD cycles. (d) Film thickness determination using AFM (for a $\mathrm{Li}_{2} \mathrm{TP}-\mathrm{NH}_{2}$ film grown with $200 \mathrm{ALD} / \mathrm{MLD}$ cycles). 

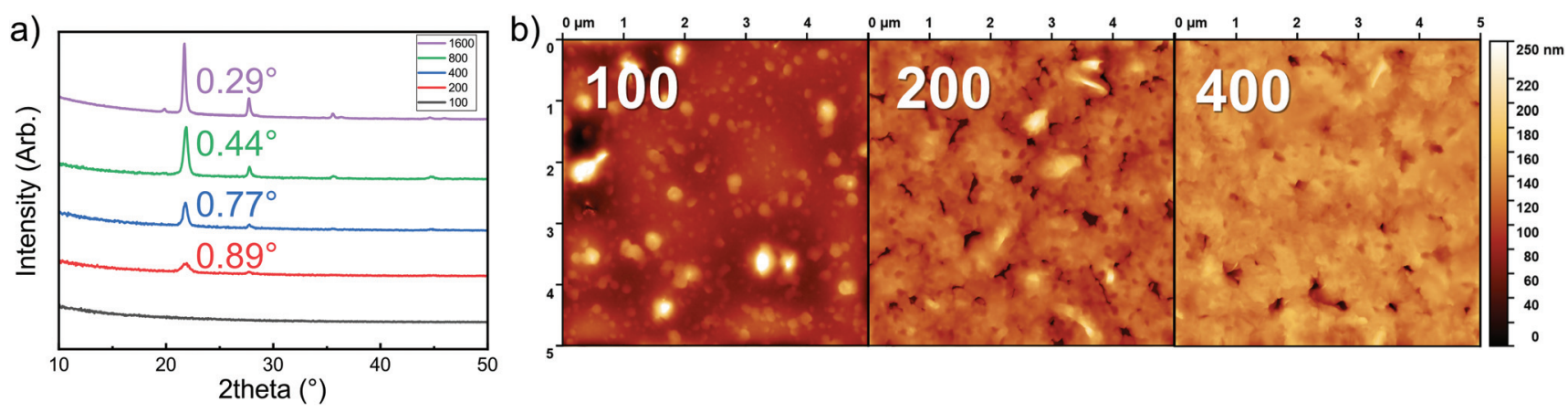

Fig. 3 (a) GIXRD patterns with calculated FWHM values, and (b) AFM images for $\mathrm{Li}_{2} \mathrm{TP}-\mathrm{NH}_{2}$ films deposited at $200{ }^{\circ} \mathrm{C}$ with increasing number of ALD/MLD cycles. The $z$-scale in AFM images is $0-50 \mathrm{~nm}$ for the sample with 100 cycles and $0-250 \mathrm{~nm}$ in samples with 200 and 400 cycles.

the main peak for representative films to demonstrate that the FWHM (full width at half maximum) decreases with an increasing number of deposition cycles, indicating an increase of the crystallite size as the films get thicker. The same observation can be made from the AFM images shown in Fig. 3b. The AFM data suggest an island-type growth mode (similar to that previously seen for the $\mathrm{Li}_{2} \mathrm{TP}$ films); ${ }^{8}$ it seems that initially the $\mathrm{Li}_{2} \mathrm{TP}-\mathrm{NH}_{2}$ films grow as amorphous, but soon islands of crystallites start to form which then serve as the preferred sites for the further film growth. This is in line with the fact that the film density (calculated from the critical angle value obtained from the XRR pattern) was initially somewhat higher, i.e. $1.59 \mathrm{~g} \mathrm{~cm}^{-3}$ for the film deposited with 100 cycles, then 1.40 and $1.44 \mathrm{~g} \mathrm{~cm}^{-3}$ for films deposited with 200 and 400 cycles, respectively.

\section{Structure of $\mathrm{Li}_{2} \mathrm{TP}_{-} \mathrm{NH}_{2}$ films}

The $\mathrm{Li}_{2} \mathrm{TP}-\mathrm{NH}_{2}$ films were crystalline, but the diffraction pattern could not be indexed according to any relevant crystal structure. It should be noted that even though the $\mathrm{Li}_{2} \mathrm{TP}-\mathrm{NH}_{2}$ phase has been previously synthesized in bulk form (plus XRD pattern is given), ${ }^{28}$ its crystal structure has not been reported. Moreover, unlike the case with e.g. the zirconium analogues, ZrTP and $\mathrm{ZrTP}-\mathrm{NH}_{2},{ }^{29}$ the $\mathrm{Li}_{2} \mathrm{TP}-\mathrm{NH}_{2}$ structure is apparently not identical with the $\mathrm{Li}_{2} \mathrm{TP}$ structure (ESI Fig. $2 \dagger$ ). We used the USPEX evolutionary algorithm ${ }^{30}$ to predict the most probable crystal structure candidates for $\mathrm{Li}_{2} \mathrm{TP}-\mathrm{NH}_{2}$. The lowestenergy structure candidate revealed through USPEX is a layered monoclinic structure with two $\mathrm{Li}_{2} \mathrm{TP}-\mathrm{NH}_{2}$ formula units in the unit cell (Fig. 4a). Interestingly, there is a direct subgroup-group relation between this $\mathrm{Li}_{2} \mathrm{TP}-\mathrm{NH}_{2}$ structure candidate $(P c)$ and the layered structure seen for $\mathrm{Li}_{2} \mathrm{TP}\left(P 2_{1} / c\right)$ in both bulk and in our ALD/MLD samples. ${ }^{8,31}$ The USPEX prediction also yielded a few other low-energy monoclinic structures for $\mathrm{Li}_{2} \mathrm{TP}-\mathrm{NH}_{2}$ with energies not much higher than in the case of the $P c$ structure (see ESI $\dagger$ ).

In Fig. $4 \mathrm{~b}$, we have indexed and LeBail fitted an experimental XRD pattern for our $\mathrm{Li}_{2} \mathrm{TP}-\mathrm{NH}_{2}$ thin-film sample; the fitting confirms that the structure must be close to the USPEXpredicted monoclinic $P c$ structure (with lattice parameters $a=$ $8.68 \AA, b=5.23 \AA, c=8.99 \AA$, and $\beta=93.76^{\circ}$ ), the monoclinic distortion being clearly seen as the splitting of the main reflection around $2 \theta \approx 22^{\circ}$. However, a closer look up indicates an additional tiny reflection around $2 \theta \approx 16^{\circ}$ (Fig. 4c), which is not explained with the $P c$ model. Considering this reflection, the best match was found for a cell with $P 2 / m$ symmetry $(a=$ $\left.8.57 \AA, b=5.28 \AA, c=8.23 \AA, \beta=99.85^{\circ}\right)$. For both the aforementioned structure models, the lattice parameters are rather
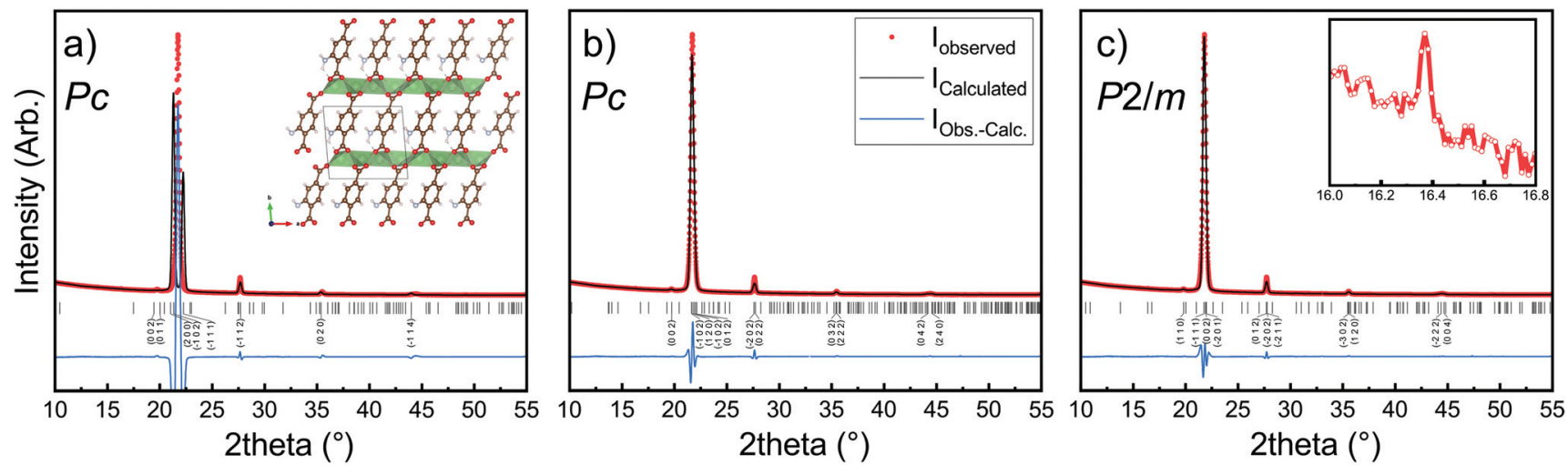

Fig. 4 XRD pattern and LeBail fits of $\mathrm{Li}_{2} \mathrm{TP}-\mathrm{NH}_{2}$ with a thickness of around $1000 \mathrm{~nm}$. In (a) the simulated profile fit using lattice parameters predicted by USPEX evolutionary algorithm, in (b) LeBail fits with tiny reflection at $16^{\circ}$ omitted, in (c) the tiny peak is included (inset). Visualization of the structure can be found in ESI. $\uparrow$ Substrate peaks are removed for clarity. 
close to the USPEX-predicted lattice, with $a=8.48 \AA$, $b=$ $5.07 \AA, c=9.16 \AA$, and $\beta=94.77^{\circ}$. It should be noted that neither the experimental nor the simulated diffraction patterns match with the previously reported pattern for a bulk $\mathrm{Li}_{2} \mathrm{TP} \mathrm{NH}_{2}$ sample. ${ }^{28}$ Steric hindrance of the amino group most likely plays a role here, in such a way that the different synthesis conditions could lead to different amino group arrangements. $^{32,33}$

The chemical bonding scheme in our $\mathrm{Li}_{2} \mathrm{TP}-\mathrm{NH}_{2}$ thin films was studied with FTIR spectroscopy. A comparison of the thinfilm spectrum to that of its TPA- $\mathrm{NH}_{2}$ precursor confirms the disappearance of the characteristic carbonyl group (only seen for the acid precursor) and the appearance of the carboxyl feature upon the metal carboxylate bond formation (ESI Fig. $3 \dagger)$. The detailed interpretation of spectral features is given in ESI Fig. $4, \dagger$ where we compare our thin-film $\mathrm{Li}_{2}$ TP-NH $\mathrm{N}_{2}$ spectrum with the previously reported FTIR spectrum for a bulk $\mathrm{Li}_{2} \mathrm{TP}-\mathrm{NH}_{2}$ sample ${ }^{28}$ and also with a spectrum calculated based on the USPEX predicted monoclinic $P c$ structure for $\mathrm{Li}_{2} \mathrm{TP}-\mathrm{NH}_{2}$; for the peak interpretation (ESI Table $2 \dagger),{ }^{34,35}$ we also considered the spectra reported for $\mathrm{Li}_{2} \mathrm{TP},{ }^{36} \mathrm{Na}_{2} \mathrm{TP}-\mathrm{NH}_{2},{ }^{33}$ and TPA-NH${ }_{2} \cdot{ }^{37}$

Most interesting observations were made by comparing the spectra of our present $\mathrm{Li}_{2} \mathrm{TP}-\mathrm{NH}_{2}$ and $\mathrm{Li}_{2} \mathrm{TP}$ thin films, see Fig. 5. The dominant peaks in terephthalate systems are caused by the asymmetric $\left(v_{\text {as }}\right)$ and symmetric $\left(v_{\mathrm{s}}\right)$ stretches of carboxylate around $1600 \mathrm{~cm}^{-1}$ and $1400 \mathrm{~cm}^{-1}$, respectively. ${ }^{14}$ The position of these peaks is often strongly affected by the nature of the substituents. In particular, electron donating groups attached to aromatic carbon skeleton are expected to shift $v_{\text {as }}$ to the higher energies (blue shift), while electron withdrawing groups such as $-\mathrm{NH}_{2}$ should result in a shift to the lower energies (red shift). Electron withdrawing substituents also shift $v_{\mathrm{s}}$ to the lower energies but this behavior is less predictable depending also on the position of the substituent

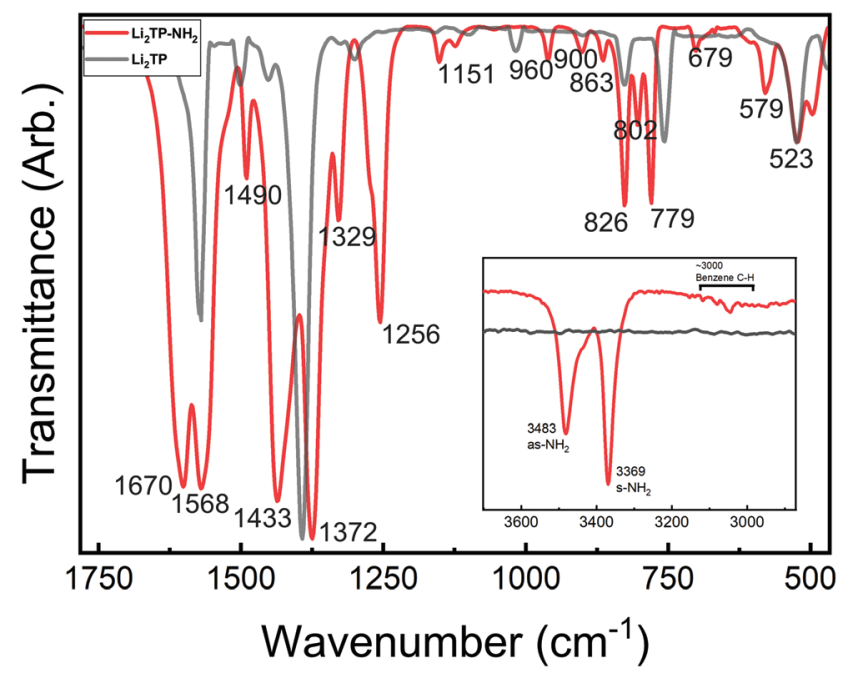

Fig. 5 FTIR spectra for $\mathrm{Li}_{2} \mathrm{TP}-\mathrm{NH}_{2}$ and $\mathrm{Li}_{2} \mathrm{TP}$; inset shows the antisymmetric (as) and symmetric (s) vibrations of the amino group at higher wavenumbers. (relative to carboxylate). ${ }^{38}$ Indeed, from Fig. 5 it is seen that $v_{\text {as }}$ red shifts from $1572 \mathrm{~cm}^{-1}\left(\mathrm{Li}_{2} \mathrm{TP}\right)$ to $1568 \mathrm{~cm}^{-1}\left(\mathrm{Li}_{2} \mathrm{TP}-\mathrm{NH}_{2}\right)$ upon the addition of the $-\mathrm{NH}_{2}$ group, and $v_{\mathrm{s}}$ from $1393 \mathrm{~cm}^{-1}$ $\left(\mathrm{Li}_{2} \mathrm{TP}\right)$ to $1372 \mathrm{~cm}^{-1}\left(\mathrm{Li}_{2} \mathrm{TP}-\mathrm{NH}_{2}\right)$. The separation $(\Delta)$ between $v_{\text {as }}$ and $v_{\mathrm{s}}$ is indicative of the binding mode of the carboxylate unit. ${ }^{14,38}$ For our $\mathrm{Li}_{2}$ TP- $\mathrm{NH}_{2}$ thin-film sample, the obtained $\Delta$ value of $196 \mathrm{~cm}^{-1}$ suggests a bridging-type connection, which is in accordance with the USPEX-predicted structure model.

Another important observation is related to the stretching modes of the benzene ring (19a and 19b; ESI Table $2 \dagger$ ), being composed of partly stretching and partly bending character of the $\mathrm{C}=\mathrm{C}$ bonds. In para disubstituted benzene (like $\mathrm{Li}_{2} \mathrm{TP}$ ) the 19a mode lies at higher wavenumbers, ${ }^{39}$ while in asymmetric trisubstituted benzene the $19 \mathrm{~b}$ mode is at a higher frequency, according to the normal coordinate analysis. ${ }^{35}$ This is important since with monosubstituted benzenes the vibration 19a has been found to become more intense with donor substituents, while $19 \mathrm{~b}$ gets stronger with acceptor substituents. A similar correlation could explain the strong peak observed at $1433 \mathrm{~cm}^{-1}$ for our $\mathrm{Li}_{2} \mathrm{TP}-\mathrm{NH}_{2}$ thin film. Another observation is that the amino group is apparently not reacting with the $\mathrm{Li}$ (thd), as the characteristic antisymmetric and symmetric peaks due to the amino group remain intact. However, this does not mean that the amino group would not show any coordination effect on the lithium oxide layer; indeed a small shoulder is seen in the asymmetric stretch of the amino peaks.

\section{Electrochemical characteristics}

Cyclic voltammetry in coin-cell configuration was used to investigate the redox behaviors of the $\mathrm{Li}_{2} \mathrm{TP}-\mathrm{NH}_{2}$ and $\mathrm{Li}_{2} \mathrm{TP}$ films. The voltammograms were recorded between $0.25-3.0 \mathrm{~V}$ for $\mathrm{Li}_{2} \mathrm{TP}-\mathrm{NH}_{2}$ and $0.01-3 \mathrm{~V}$ for $\mathrm{Li}_{2} \mathrm{TP}$; in the first case scanning to too low potentials was found to be detrimental and was hence avoided. The $1^{\text {st }}$ and $2^{\text {nd }}$ cycles were measured with a scan rate of $0.1 \mathrm{mV} \mathrm{s}^{-1}$, followed by cycles with higher rates of 0.5 and $1 \mathrm{mV} \mathrm{s}^{-1}$ (Fig. 6). For both materials, the $1^{\text {st }}$ cycle was found drastically different from the following cycles, presumably due to solid electrolyte interphase (SEI) formation. ${ }^{40}$ After the $1^{\text {st }}$ cycle, $\mathrm{Li}_{2} \mathrm{TP}-\mathrm{NH}_{2}$ shows wide reduction and oxidation peaks centered around 0.62 and $0.82 \mathrm{~V}$, respectively,
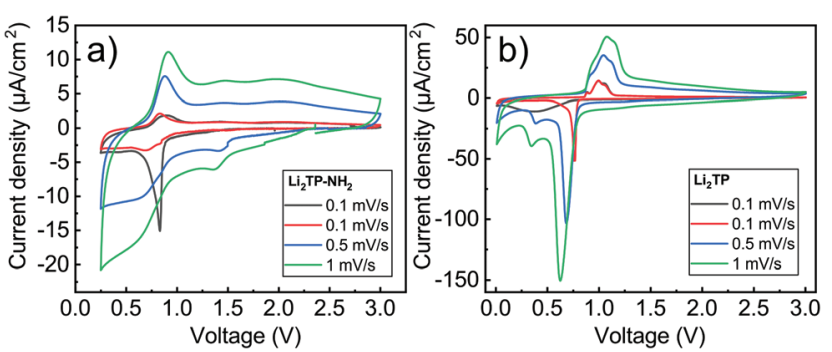

Fig. 6 Cyclic voltammograms for (a) $\mathrm{Li}_{2} \mathrm{TP}-\mathrm{NH}_{2}(0.25-3.0 \mathrm{~V}$ ), and (b) $\mathrm{Li}_{2} \mathrm{TP}(0.01-3.0 \mathrm{~V})$ with various scan rates. The dark grey line represents the $1^{\text {st }}$ cycle, and the red, blue, and green lines represent the subsequent cycles. Each cycle starts at the original open circuit voltage, being ca. 2 $\checkmark$ for both the samples. 
while $\mathrm{Li}_{2} \mathrm{TP}$ exhibits relatively sharp peaks at $0.76 \mathrm{~V}$ (reduction) and $1.0 \mathrm{~V}$ (oxidation). Hence the electron-donating amino group indeed lowers the redox peak potential of $\mathrm{Li}_{2} \mathrm{TP}$ by 0.14 $\mathrm{V}$. The magnitude of the decrease in the potential is not enormous but it is in line with the results previously reported for the Na-based counterparts $(0.19 \mathrm{~V}$; quasi-open circuit voltages). ${ }^{24}$ According to DFT calculations by Renault et $a .^{28}$ lithiation of $\mathrm{Li}_{2} \mathrm{TP}-\mathrm{NH}_{2}$ occurs preferably on the carbonyl next to the amino group. This preferred process can be observed as a shoulder of the main reduction peak; this shoulder is however seen with the slowest scan rate of $0.1 \mathrm{mV} \mathrm{s}^{-1}$ only, indicating slow diffusion or kinetics of the electrode (Fig. 6a; red curve). Finally we note that the positive impact of the amino group in lowering the redox potential loses some of its significance due to the poorer electrode kinetics and/or slower diffusion into the electrode, the reason of which will be discussed in more detail later on.

Galvanostatic charge/discharge cyclings were performed with various current rates to investigate the cycling performance (Fig. 7); in these experiments, the voltage range was 0.4-3.0 $\mathrm{V}$ for both materials. In line with the cyclic voltammetry results, the performance of $\mathrm{Li}_{2} \mathrm{TP}-\mathrm{NH}_{2}$ was inferior to that of $\mathrm{Li}_{2} \mathrm{TP}$ in the rate capability tests. The capacity retention between 1.1 and $53.0 \mu \mathrm{A} \mathrm{cm}^{-2}$ was $67 \%$ for $\mathrm{Li}_{2} \mathrm{TP}$ but only $24 \%$ for $\mathrm{Li}_{2} \mathrm{TP}-\mathrm{NH}_{2}$. After the initial cycles, the coulombic efficiency on average was $99 \%$ for $\mathrm{Li}_{2} \mathrm{TP}$ and $97 \%$ for $\mathrm{Li}_{2} \mathrm{TP}-\mathrm{NH}_{2}$. The capacity values were $\sim 9 \mu \mathrm{A} \mathrm{h} \mathrm{cm}{ }^{-2}$ for $\mathrm{Li}_{2} \mathrm{TP}$ and $\sim 8 \mu \mathrm{A} \mathrm{h} \mathrm{cm} \mathrm{cm}^{-2}$ for $\mathrm{Li}_{2} \mathrm{TP}-\mathrm{NH}_{2}$.

While the targeted role of the additional functional groups is to decrease or increase the redox potential, ${ }^{41}$ they may also have unintended consequences. It has been shown that electron donor functional groups may disturb the $\pi-\pi$ orbital stacking of the benzene rings and actually decrease the conductivity of the material. ${ }^{42}$ According to the calculations by Zhang et al., ${ }^{43} \mathrm{Li}^{+}$hopping is fast in $\mathrm{Li}_{2} \mathrm{TP}$ and occurs mostly within the stacked $\pi$-orbitals of the benzene rings; this is also the path of the least resistance for the electrons in $\mathrm{Li}_{2} \mathrm{TP} .{ }^{19,44}$ Upon replacing one of the hydrogen atoms in the benzene ring with an additional functional group a shift occurs for the HOMO and LUMO orbitals and thereby also for the $\pi$-orbital stacking. This might be one of the reasons for the poorer electrochemical performance. Functional groups may also introduce a steric hindrance for the $\mathrm{Li}^{+}$diffusion.

The flat (dis)charge curve extending even up to high current densities for $\mathrm{Li}_{2} \mathrm{TP}$ is not seen for $\mathrm{Li}_{2} \mathrm{TP}-\mathrm{NH}_{2}$ (Fig. 7). Such sloping potentials as observed for the present $\mathrm{Li}_{2} \mathrm{TP}-\mathrm{NH}_{2}$ thin film and also previously reported for bulk $\mathrm{Li}_{2} \mathrm{TP}-\mathrm{NH}_{2} \cdot{ }^{28}$ Sloping voltage profiles are rather common in organic polymer batteries, where the redox active groups are interconnected and the potential strongly depends on the lithiation degree. ${ }^{45}$ Park et al. ${ }^{24}$ explained the difference as a change of the reaction type, from two-phase reaction to one-phase reaction. Electron donors are prone to cause charge reorganizations and formation of occupied states in the aromatic core; these changes may have an effect on the voltage profile. ${ }^{46}$ Therefore, there might be multiple reasons behind the observed change in the voltage profile.

From the rate capability measurement data shown in Fig. 7 it can be seen that the delithiation is never complete in the cycle succeeding the increase in current density, i.e., in these first discharge/charge cycles the capacity from lithiation is larger than the capacity from delithiation indicating that with the same current density, delithiation is the slower reaction. Afterwards, the capacity loss can be recovered with lower current rates, as seen in the increased delithiation capacity. A larger cut-off voltage for delithiation might solve the issue, but $3.0 \mathrm{~V}$ is already a very high voltage for a negative electrode material.

Its has been observed that the electronic conductivity of organic electrodes increases with the amount of intercalated lithium. ${ }^{44}$ Therefore, during lithiation, the material probably becomes more and more conducting such that it becomes easier to intercalate lithium to the lattice. This is actually what can be seen with $\mathrm{Li}_{2} \mathrm{TP}$ with the larger current densities during lithiation where the potential momentarily drops lower than the plateau. Hence, after a certain lithiation threshold, it becomes easier to continue the lithiation, presumably due to the decrease in resistivity. Clearly, this same behavior is not seen with $\mathrm{Li}_{2} \mathrm{TP}-\mathrm{NH}_{2}$, presumably due to its intrinsically higher resistivity.

The performance of the films was also tested over 200 charge/discharge cycles. Cycling started again with a slow
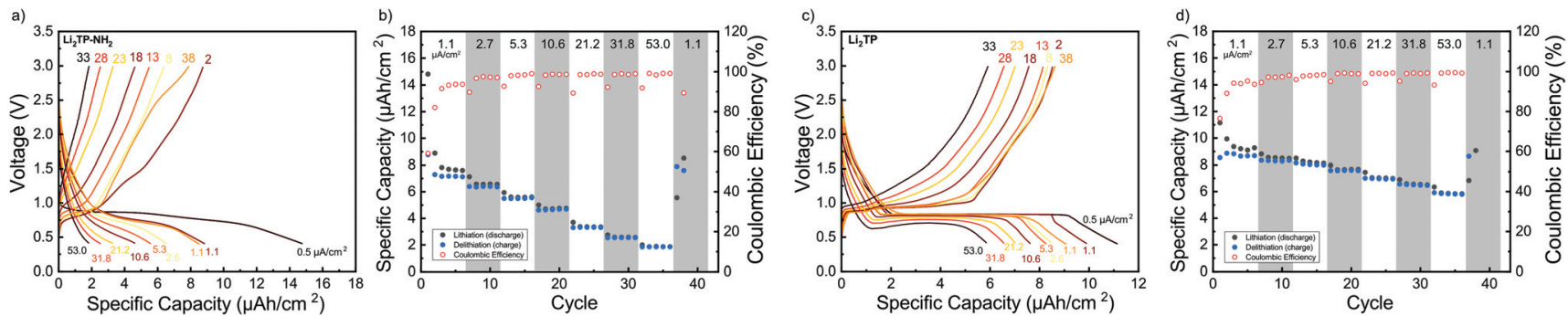

Fig. 7 Rate capability measurements for $\mathrm{Li}_{2} \mathrm{TP}-\mathrm{NH}_{2}$ (a and b) and $\mathrm{Li}_{2} \mathrm{TP}$ (c and d): cycling starts with a format cycle with a small current of $0.5 \mu \mathrm{A}$ $\mathrm{cm}^{-2}$, thereafter the current is increased after every 5 cycles before returning to the original value $\left(1.1,2.7,5.3,10.6,21.2,31.8,53.0 \mu \mathrm{A} \mathrm{cm}{ }^{-2}\right)$. In (a) and (c) the shape of the charge-discharge curve at different cycles and in (b) and (d) capacity plotted with coulombic efficiency. 

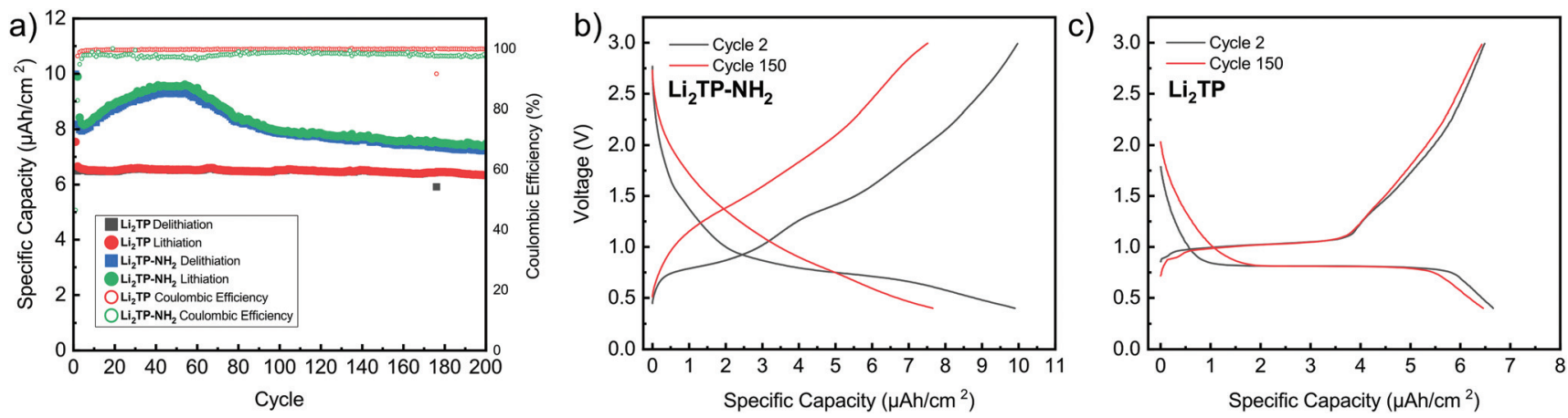

Fig. 8 Performance of $\mathrm{Li}_{2} \mathrm{TP}-\mathrm{NH}_{2}$ and $\mathrm{Li}_{2} \mathrm{TP}$ during extended cycling in a half-cell. In (a) capacity retention and coulombic efficiency over 200 cycles and in (b) and (c) the observed change in the charge-discharge curve of $\mathrm{Li}_{2} \mathrm{TP}-\mathrm{NH}_{2}$ and $\mathrm{Li}{ }_{2} \mathrm{TP}$, respectively.

format cycle $\left(0.5 \mu \mathrm{A} \mathrm{cm} \mathrm{cm}^{-2}\right)$ followed by cycling with $2.7 \mu \mathrm{A}$ $\mathrm{cm}^{-2}$ (Fig. 8). It was seen that $\mathrm{Li}_{2} \mathrm{TP}$ retains $95 \%$ of its capacity and shows very stable performance over the whole cycling period, while $\mathrm{Li}_{2} \mathrm{TP}-\mathrm{NH}_{2}$ retains $90 \%$ of its capacity, but also shows an interesting behavior, where the capacity first increases followed by a steady decrease (Fig. 8a). In previous works, similar abnormal excess capacity has been ascribed to the lithiation of the aromatic skeleton. This could have an effect on the reversibility of the redox reaction as some of the lithium inserted in low potentials would require very high potentials to leave the electrode. ${ }^{47}$ Amino groups have been reported to suppress the dissolution of organic electrodes, ${ }^{48}$ so the decrease in capacity is most likely not due to dissolution, and as can be seen in the Fig. 9b, the thickness of the film has only increased due to the formation of SEI. The coulombic efficiency of $\mathrm{Li}_{2} \mathrm{TP}$ is very high (>99\%) after the initial cycles compared to rather poor efficiency of $95 \%$ of $\mathrm{Li}_{2} \mathrm{TP}-\mathrm{NH}_{2}$. The lower coulombic efficiency is a direct sign that the redox reaction with $\mathrm{Li}_{2} \mathrm{TP}-\mathrm{NH}_{2}$ is not as reversible as with $\mathrm{Li}_{2} \mathrm{TP}$ within this potential range. The coulombic efficiency of $\mathrm{Li}_{2} \mathrm{TP}-\mathrm{NH}_{2}$ is noticeably low during the cycles related to the lithiation of the aromatic skeleton. Similar low coulombic efficiency with $\mathrm{Li}_{2} \mathrm{TP}$ was observed by Lee et $a l^{47}$ when the low potential cut-off voltage was decreased to $0.0 \mathrm{~V}$, where the aromatic skeleton of $\mathrm{Li}_{2} \mathrm{TP}$ was purposely lithiated. Therefore, both the gradual increase in capacity and the lower coulombic efficiency are possibly caused by the lithiation of the aromatic skeleton.

The shape of the charge-discharge curve of $\mathrm{Li}_{2} \mathrm{TP}-\mathrm{NH}_{2}$ changes drastically over the cycling while that of $\mathrm{Li}_{2} \mathrm{TP}$ remains essentially unchanged (Fig. $8 \mathrm{~b}$ and c). The steady slope of $\mathrm{Li}_{2} \mathrm{TP}-\mathrm{NH}_{2}$ disappears completely and the over-potential increases. The drastic changes in the shape of the chargedischarge curve are a direct indication of changes occurring on the material during cycling, which might be due to the amorphization of the material caused by the lithiation of the aromatic skeleton ${ }^{47}$ or other significant morphological changes of the electrode. In Fig. 9 we display post-mortem XRD and cross section SEM image for a pristine and cycled electrodes; both clearly show that the $\mathrm{Li}_{2} \mathrm{TP}-\mathrm{NH}_{2}$ turns amorphous upon cycling. From the SEM images it is moreover seen that the cycled electrode is considerably thicker (formation of SEI) and it consists of a sea of small particles, while in the pristine electrode the crystallites are larger. The $\mathrm{Li}_{2} \mathrm{TP}$ on the otherhand shows only minor changes on the surface and the crystallites remain intact.

Material properties such as the electrode morphology ${ }^{49}$ and shape of crystallites ${ }^{50,51}$ of the active material may have a considerable effect on mass and electron transport of the electrode and thereby its electrochemical performance. In addition, it has been shown that particle pulverization can a)

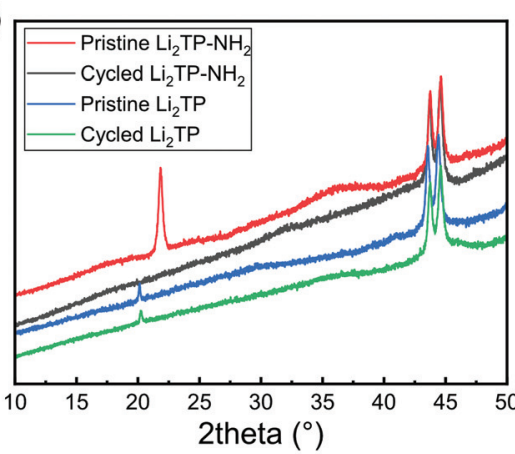

b)

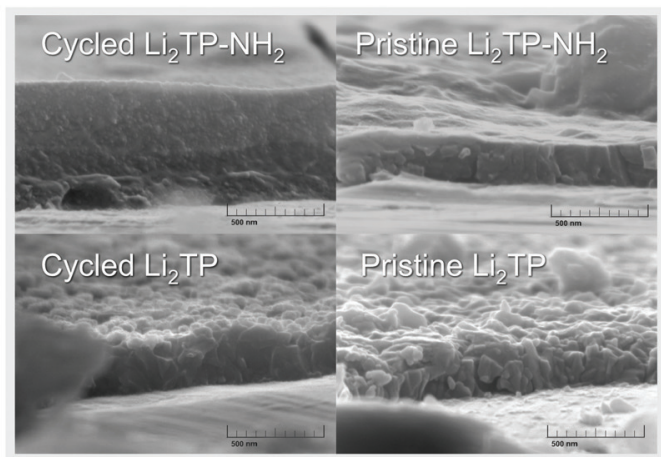

Fig. 9 (a) Diffraction patterns of $\mathrm{Li}_{2} \mathrm{TP}-\mathrm{NH}_{2}$ (the shift in baseline and peaks at $44^{\circ}$ are due to steel substrate), and (b) SEM images for a pristine and cycled $\mathrm{Li}_{2} \mathrm{TP}-\mathrm{NH}_{2}$ and $\mathrm{Li}_{2} \mathrm{TP}$ electrodes. 
take place and is detrimental to the electrochemical performance of organic electrode materials. ${ }^{52}$ The observed morphological changes occurring during cycling for our $\mathrm{Li}_{2} \mathrm{TP}-\mathrm{NH}_{2}$ electrode are very likely the cause of the capacity decay. We tentatively believe that the breakage of the crystal structure originates from the lithiation of the aromatic skeleton. ${ }^{47}$

\section{Conclusions}

We have widened the repertoire of lithium-bearing ALD/MLD processes to the amino-functionalized lithium terephthalate, i.e. dilithium 2-aminoterephthalate or $\mathrm{Li}_{2} \mathrm{TP}^{-} \mathrm{NH}_{2}$. The $\mathrm{Li}_{2} \mathrm{TP}-\mathrm{NH}_{2}$ films were found to initially grow smooth and amorphous but after a certain threshold number of ALD/MLD cycles crystallites started to form. The $\mathrm{Li}_{2} \mathrm{TP}-\mathrm{NH}_{2}$ crystals were not of any previously known crystal structure; we thus determined the structure with a combined prediction (USPEX), modelling (DFT) and fitting (LeBail) approach. Additional evidence for the resulting monoclinic structure was revealed from the FTIR data.

Electrochemical measurements verified the validity of our initial design concept, that is, the possibility to use additional electron-donating functional groups to lower the redox potential of the lithium terephthalate $\mathrm{Li}_{2} \mathrm{TP}$ anode material. The decrease in the potential achieved with the amino group attached to the terephthalate backbone was 0.14 $\mathrm{V}$. Unfortunately, this benefit was gained at the expense of the lower coulombic efficiency and poorer rate capability. We observed effects such as morphology, crystallinity changes, and delamination but no dissolution in the post-mortem analysis of the electrodes.

As these thin film electrodes are free from any conductive carbon or other additives and their preparation route is the same their performance should be indicative of the actual material. This especially makes the post-mortem analysis simpler as the interactions in the cells are minimized. We believe that manufacturing the organic electrode materials in the thin film form is the key to better understand their electrochemical performance.

\section{Experimental}

The $\mathrm{Li}_{2} \mathrm{TP}-\mathrm{NH}_{2}$ and $\mathrm{Li}_{2} \mathrm{TP}$ (for reference) thin films were deposited from $\mathrm{Li}$ (thd) (thd $=2,2,6,6$-tetramethyl-3,5-heptanedione), 2-aminoterephthalic acid (98\%, Tokyo Chemical Industry Co. Ltd) and terephthalic acid (98\%, Tokyo Chemical Industry Co. Ltd) precursors. The Li(thd) precursor powder was synthetized by mixing LiOH $\left(98 \%\right.$, Alfa Aesar) in $1: 1 \mathrm{H}_{2} \mathrm{O}$ and EtOH mixture with thd (Tokyo Chemical Industry Co. Ltd) in $1: 1 \mathrm{H}_{2} \mathrm{O}$ and $\mathrm{EtOH}$ mixture. The resulting white mixture was further dried in vacuum and purified by sublimation. The films were deposited in an F-120 flow-type hot-wall ALD reactor (ASM Microchemistry Ltd) at low pressure ( $\sim 5$ mbar). The carrier and purging gas was nitrogen (produced from the air with nitrogen generator Parker HPN2-5000, with less than $10 \mathrm{ppm}$ of oxygen). For the $\mathrm{Li}_{2} \mathrm{TP}$ films, the deposition process parameters were adopted from our previous work, ${ }^{8}$ while for the $\mathrm{Li}_{2} \mathrm{TP}-\mathrm{NH}_{2}$ films the parameters were optimized in this work. The sublimation temperature for the TPA- $\mathrm{NH}_{2}$ was $185{ }^{\circ} \mathrm{C}$ and $175{ }^{\circ} \mathrm{C}$ for $\mathrm{Li}($ thd). For the structure characterizations, $\operatorname{Si}(100)$ substrates were used, while for the electrochemical characterizations the films were grown on stainless steel disks $(\varnothing 15.5 \mathrm{~mm})$.

X-ray diffraction patterns were collected for the films with PANanalytical X'Pert Pro diffractometer $\left(\mathrm{Cu} \quad \mathrm{K} \alpha_{1} ; \lambda=\right.$ $1.540598 \AA$ ) both in the conventional (XRD) and the grazingincidence (GIXRD; incident angle $0.5^{\circ}$ ) modes. The XRD patterns measured in Bragg-Brentano mode were indexed and fitted with the LeBail method using the options integrated into the FullProf software package (Treor90 and CheckCell). ${ }^{53}$ The film thickness and density were estimated for the films from the X-ray reflection (XRR) patterns measured with the same apparatus. It should be noted that the density calculation (see ESI $\dagger$ ) is based on an approximated chemical composition of the film, which naturally courses some uncertainty; nevertheless, within a series of similar samples, the possible trends should be meaningful. ${ }^{8,54}$ For the thickest and roughest films, the film thickness was determined with spectroscopic ellipsometer measurements (Woollam Spectroscopic Ellipsometer; CompleteEASE software).

The bonding structure and the presence of intended functional groups were investigated with Fourier transform infrared spectroscopy (FTIR; Nicolet Magna 750). The FTIR measurements were performed in a range of 400 to $4000 \mathrm{~cm}^{-1}$ with a resolution of $4 \mathrm{~cm}^{-1}$. The atomic force microscope images were conducted in tapping mode with a Veeco Dimension 5000. Tips used were Mikromasch HQ: NSC14/AlBS tips with a typical radius of $8 \mathrm{~nm}$ and $5 \mathrm{~N} \mathrm{~m}^{-1}$ force constant.

For the electrochemical evaluation, the thin films were deposited on steel substrates. The thickness of the thin film could not be measured from the steel substrate, and therefore the exact mass of the electrode cannot be reliably determined. However, the mass of the electrode can be estimated from the growth rate on the silicon substrate. This will cause some systematic error in the data because the nucleation model on different substrates can differ. ${ }^{55}$ However, as the film's goal thickness was fixed to $170 \mathrm{~nm}$, it makes much more sense just to compare the capacity per area and it makes the electrode comparison more practical. The theoretical capacity of $\mathrm{Li}_{2} \mathrm{TP}^{-\mathrm{NH}_{2}}$ is $277.8 \mathrm{~mA} \mathrm{~h} \mathrm{~g}{ }^{-1}$. Assuming the film's thickness of $170 \mathrm{~nm}$ and calculated density, the areal capacity should be around $8 \mu \mathrm{A} \mathrm{h} \mathrm{cm} \mathrm{cm}^{-2}$ for both of the materials. This is relatively close value to the measured ones when taking into account all of the possible errors in calculated density, film thickness, and the exact area of the current collector as films grow in every direction and underneath the substrate. The samples were first dried in a vacuum oven $\left(110^{\circ} \mathrm{C}\right)$ for 24 hours and moved into argon filled glovebox with oxygen levels under $1 \mathrm{ppm}$. Afterwards, the films were directly applied as the working electrode in a CR2016 coin cell with a lithium metal counter elec- 
trode to evaluate their electrochemical performance. The electrolyte was $1 \mathrm{M} \mathrm{LiPF}_{6}$ in a 1:1 EC/DMC solution. Cyclic voltammetry measurements were carried out with Autolab PGSTA302N potentiostat, and galvanostatic cyclings in a Neware battery testing unit with various current densities. The galvanostatic cycling voltage range was chosen to limit the reactions occurring below $0.4 \mathrm{~V}$. In cyclic voltammetry each cycle starts at the original open circuit voltage, being ca. $2 \mathrm{~V}$ for both the samples.

We also carried out crystal structure predictions for $\mathrm{Li}_{2} \mathrm{TP}-\mathrm{NH}_{2}$ with the evolutionary algorithms implemented in the USPEX 9.4.4 code. ${ }^{30}$ All quantum chemical calculations within the USPEX simulations were performed using Quantum Espresso program package (version 6.0). ${ }^{56}$ We used density functional theory (DFT) with the PBE exchange-correlation functional and GBRV ultrasoft pseudopotentials. ${ }^{57,58}$ Kinetic energy cut-offs of 40 Ry and 200 Ry were applied for wavefunctions and charge densities, respectively. In the USPEX simulations, we applied a molecular crystal structure prediction algorithm. ${ }^{59}$ Each simulation included two $\mathrm{Li}^{+}$cations per each 2-aminoterephtalate dianion and all structures where the dianion did not stay intact were discarded. The USPEX simulations were run with different numbers of formula units in the unit cell $(Z=2,3$, and 4$)$. All simulations produced a similar type of layered structures, but the lowest-energy structures were obtained from two simulations carried out for $Z=2$. The full USPEX and Quantum Espresso input files for one $Z=$ 2 simulation are included in ESI. $\dagger$

The low-energy structures produced by USPEX were re-optimized using the CRYSTAL17 program package, hybrid PBE0 density functional method, and triple-zeta-valence + polarization (TZVP) level of basis set. The Gaussian Type Orbital basis sets have been derived from Karlsruhe basis sets ${ }^{60}$ and adapted for solid-state calculations. ${ }^{13,61}$ For all low-energy structures, we applied a $k$-mesh of $3 \times 5 \times 3$ for the reciprocal space sampling. The structures were optimized with the default optimization convergence criteria in CRYSTAL17 and the lowest-energy structures were confirmed to be true local minima by means of harmonic frequency calculations. The harmonic vibrational frequencies and IR intensities were obtained with the computational scheme implemented in CRYSTAL. $^{62}$ For the simulated IR spectra, we applied a Lorentzian lineshape and an FWHM of $16 \mathrm{~cm}^{-1}$. The band assignments were carried out by visual inspection of the normal modes using the Jmol program package. ${ }^{63}$

\section{Conflicts of interest}

There are no conflicts to declare.

\section{Acknowledgements}

We acknowledge the funding from European Research Council under the European Union's Seventh Framework Programme
(FP/2007-2013)/ERC Advanced Grant Agreement (339478) and Academy of Finland (296299), and the use of the RawMatTERS Finland Infrastructure (RAMI) and OtaNano - Nanomicroscopy Center (Aalto-NMC) at Aalto University. Computational resources were provided by CSC-the Finnish IT Center for Science.

\section{Notes and references}

1 M. Armand and J.-M. Tarascon, Nature, 2008, 451, 652-657.

2 J. Heiska, M. Nisula and M. Karppinen, J. Mater. Chem. A, 2019, 7, 18735-18758.

3 T. B. Schon, B. T. McAllister, P.-F. Li and D. S. Seferos, Chem. Soc. Rev., 2016, 45, 6345-6404.

4 Y. Liang and Y. Yao, Joule, 2018, 2, 1690-1706.

5 Q. Zhao, Y. Lu and J. Chen, Adv. Energy Mater., 2017, 7, 1601792.

6 Z. Zhu and J. Chen, J. Electrochem. Soc., 2015, 162, 23932405.

7 Y. Liang, P. Zhang, S. Yang, Z. Tao and J. Chen, Adv. Energy Mater., 2013, 3, 600-605.

8 M. Nisula and M. Karppinen, Nano Lett., 2016, 16, 1276-1281.

9 M. Nisula and M. Karppinen, J. Mater. Chem. A, 2018, 6, 7027-7033.

10 S. M. George, Chem. Rev., 2010, 110, 111-131.

11 A. S. Asundi, J. A. Raiford and S. F. Bent, ACS Energy Lett., 2019, 4, 908-925.

12 O. Nilsen, K. Klepper, H. Nielsen and H. Fjellvåg, ECS Trans., 2008, 16, 3-14.

13 M. Nisula, J. Linnera, A. J. Karttunen and M. Karppinen, Chem. - Eur. J., 2017, 23, 2988-2992.

14 J. Penttinen, M. Nisula and M. Karppinen, Chem. - Eur. J., 2017, 23, 18225-18231.

15 E. Ahvenniemi and M. Karppinen, Chem. Commun., 2016, 52, 1139-1142.

16 P. Sundberg and M. Karppinen, Beilstein J. Nanotechnol., 2014, 5, 1104-1136.

17 J. Penttinen, M. Nisula and M. Karppinen, Chem. - Eur. J., 2019, 25, 11466-11473.

18 A. Khayyami and M. Karppinen, Chem. Mater., 2018, 30, 5904-5911.

19 M. Armand, S. Grugeon, H. Vezin, S. Laruelle, P. Ribière, P. Poizot and J.-M. Tarascon, Nat. Mater., 2009, 8, 120-125.

20 L. Zhao, J. Zhao, Y. S. Hu, H. Li, Z. Zhou, M. Armand and L. Chen, Adv. Energy Mater., 2012, 2, 962-965.

21 X. Yan, C.-Y. Fan, X. Yang, Y.-Y. Wang, B.-H. Hou, W.-L. Pang and X.-L. Wu, Mater. Today Energy, 2019, 13, 302-307.

22 F. Wan, X.-L. Wu, J.-Z. Guo, J.-Y. Li, J.-P. Zhang, L. Niu and R.-S. Wang, Nano Energy, 2015, 13, 450-457.

23 X. Yan, H. Ye, X.-L. Wu, Y.-P. Zheng, F. Wan, M. Liu, X.-H. Zhang, J.-P. Zhang and Y.-G. Guo, J. Mater. Chem. A, 2017, 5, 16622-16629.

24 Y. Park, D.-S. Shin, S. H. Woo, N. S. Choi, K. H. Shin, S. M. Oh, K. T. Lee and S. Y. Hong, Adv. Mater., 2012, 24, 3562-3567. 
25 X. Meng, J. Mater. Chem. A, 2017, 5, 18326-18378.

26 A. Khayyami, A. Philip and M. Karppinen, Angew. Chem., Int. Ed., 2019, 58, 13400-13404.

27 O. Filies, O. Böling, K. Grewer, J. Lekki, M. Lekka, Z. Stachura and B. Cleff, Appl. Surf. Sci., 1999, 141, 357365.

28 S. Renault, V. A. Oltean, M. Ebadi, K. Edström and D. Brandell, Solid State Ionics, 2017, 307, 1-5.

29 L. Shen, S. Liang, W. Wu, R. Liang and L. Wu, Dalton Trans., 2013, 42, 13649.

30 C. W. Glass, A. R. Oganov and N. Hansen, Comput. Phys. Commun., 2006, 175, 713-720.

31 J. a. Kaduk, Acta Crystallogr., Sect. B: Struct. Sci., 2000, 56, 474-485.

32 K. B. Lausund, V. Petrovic and O. Nilsen, Dalton Trans., 2017, 46, 16983-16992.

33 J. Sienkiewicz-Gromiuk, L. Mazur, A. Bartyzel and Z. Rzączyńska, J. Inorg. Organomet. Polym. Mater., 2012, 22, 1325-1331.

34 P. Larkin, Infrared and Raman Spectroscopy, Elsevier, 2011, vol. 9.

35 G. Varsányi, in Vibrational Spectra of Benzene Derivatives, Elsevier, 1969, pp. 141-393.

36 M. Fathima Beegum, L. Usha Kumari, B. Harikumar, H. T. Varghese and C. Yohannan Panicker, Rasayan J. Chem., 2008, 1, 117-124.

37 M. Karabacak, M. Cinar, Z. Unal and M. Kurt, J. Mol. Struct., 2010, 982, 22-27.

38 M. B. Hay and S. C. B. Myneni, Geochim. Cosmochim. Acta, 2007, 71, 3518-3532.

39 J. F. Arenas and J. I. Marcos, Spectrochim. Acta, Part A, 1979, 35, 355-363.

40 V. A. Oltean, B. Philippe, S. Renault, R. Félix Duarte, H. Rensmo and D. Brandell, Chem. Mater., 2016, 28, 87428751.

41 Y. Lu, Q. Zhang, L. Li, Z. Niu and J. Chen, Chem, 2018, 4, 2786-2813.

42 S. E. Wheeler, J. Am. Chem. Soc., 2011, 133, 10262-10274.

43 Y. Y. Zhang, Y. Y. Sun, S. X. Du, H.-J. Gao and S. B. Zhang, Appl. Phys. Lett., 2012, 100, 1-4.

44 N. Ogihara, N. Ohba and Y. Kishida, Sci. Adv., 2017, 3, e1603103.

45 S. Muench, A. Wild, C. Friebe, B. Häupler, T. Janoschka and U. S. Schubert, Chem. Rev., 2016, 116, 9438-9484.

46 J. Lüder, F. Legrain, Y. Chen and S. Manzhos, MRS Commun., 2017, 7, 523-540.
47 H. H. Lee, Y. Park, K. H. Shin, K. T. Lee and S. Y. Hong, ACS Appl. Mater. Interfaces, 2014, 6, 19118-19126.

48 B. Tian, Z. Ding, G.-H. Ning, W. Tang, C. Peng, B. Liu, J. Su, C. Su and K. P. Loh, Chem. Commun., 2017, 53, 2914-2917.

49 S. Wang, L. Wang, K. Zhang, Z. Zhu, Z. Tao and J. Chen, Nano Lett., 2013, 13, 4404-4409.

50 N. Ogihara and Y. Kishida, Commun. Electrochem., 2015, 83, 861-863.

51 L. Fédèle, F. Sauvage, J. Bois, J. M. Tarascon and M. Bécuwe, J. Electrochem. Soc., 2014, 161, 46-52.

52 C. Luo, Y. Zhu, Y. Xu, Y. Liu, T. Gao, J. Wang and C. Wang, J. Power Sources, 2014, 250, 372-378.

53 J. Rodríguez-Carvajal, Physica B: Condens. Matter, 1993, 192, 55-69.

54 V. Holý, P. Ullrich and B. Tilo, High Resolution X-Ray Scattering from Thin Films and Multilayers, Springer-Verlag, Berlin, 1999.

55 R. L. Puurunen and W. Vandervorst, J. Appl. Phys., 2004, 96, 7686-7695.

56 P. Giannozzi, O. Andreussi, T. Brumme, O. Bunau, M. Buongiorno Nardelli, M. Calandra, R. Car, C. Cavazzoni, D. Ceresoli, M. Cococcioni, N. Colonna, I. Carnimeo, A. Dal Corso, S. de Gironcoli, P. Delugas, R. A. DiStasio, A. Ferretti, A. Floris, G. Fratesi, G. Fugallo, R. Gebauer, U. Gerstmann, F. Giustino, T. Gorni, J. Jia, M. Kawamura, H.-Y. Ko, A. Kokalj, E. Küçükbenli, M. Lazzeri, M. Marsili, N. Marzari, F. Mauri, N. L. Nguyen, H.-V. Nguyen, A. Oterode-la-Roza, L. Paulatto, S. Poncé, D. Rocca, R. Sabatini, B. Santra, M. Schlipf, A. P. Seitsonen, A. Smogunov, I. Timrov, T. Thonhauser, P. Umari, N. Vast, X. Wu and S. Baroni, J. Phys.: Condens. Matter, 2017, 29, 465901.

57 J. P. Perdew, K. Burke and M. Ernzerhof, Phys. Rev. Lett., 1996, 77, 3865-3868.

58 K. F. Garrity, J. W. Bennett, K. M. Rabe and D. Vanderbilt, DOI: 10.1016/j.commatsci.2013.08.053.

59 Q. Zhu, A. R. Oganov, C. W. Glass and H. T. Stokes, Acta Crystallogr., Sect. B: Struct. Sci., 2012, 68, 215-226.

60 F. Weigend and R. Ahlrichs, Phys. Chem. Chem. Phys., 2005, 7, 3297.

61 A. J. Karttunen, T. Tynell and M. Karppinen, J. Phys. Chem. C, 2015, 119, 13105-13114.

62 F. Pascale, C. M. Zicovich-Wilson, F. López Gejo, B. Civalleri, R. Orlando and R. Dovesi, J. Comput. Chem., 2004, 25, 888-897.

63 Jmol: an open-source Java viewer for chemical structures in 3D. http://www.jmol.org/. 